\section{Ce que les planaires nous apprennent sur les cils, les centrioles et les centrosomes}

Juliette Azimzadeh
University of California, Department of Biochemistry and Biophysics, 600 16th street, San Francisco, CA 94158, États-Unis. juliette.azimzadeh@ucsf.edu

\section{Rôles physiologiques des cils cellulaires}

Les cils sont de longues protrusions que l'on trouve pratiquement sur toutes nos cellules et qui, en fonctionnant comme des antennes pour la réception de signaux intercellulaires, jouent un rôle majeur dans le développement et l'homéostasie des vertébrés (Figure 1A). Les cils peuvent aussi être motiles et intervenir, par exemple, dans la propulsion des spermatozoïdes ou l'évacuation du mucus dans les voies respiratoires. Chez l'humain, des mutations qui affectent l'assemblage des cils sont à l'origine d'une classe de pathologies appelées ciliopathies. Celles-ci peuvent consister en des anomalies du développement comme l'hydrocéphalie ou la polykystose rénale, ou encore s'accompagner d'infections respiratoires chroniques et de problèmes de fertilité [1].

Les cils sont assemblés à partir des centrioles, des organites cylindriques constitués d'une grande diversité de protéines dont beaucoup n'ont pas encore été caractérisées. Outre leur rôle dans l'assemblage des cils, les centrioles forment aussi le cœur du centrosome, le principal centre organisateur du cytosquelette microtubulaire de la cellule animale (Figure 1B). Dans les cellules en prolifération, les deux centrioles présents au sein du centrosome sont dupliqués à chaque cycle cellulaire pour former deux centrosomes, qui participent à la séparation des chromosomes, et dont hérite chacune des deux cellules issues de la division. Ces dernières années, plusieurs protéines contrôlant les étapes initiales de la duplication des centrioles, comme la kinase Plk4 (polo kinase 4) et les protéines Sas-4 ou Sas-6, ont pu être identifiées [2, 3].

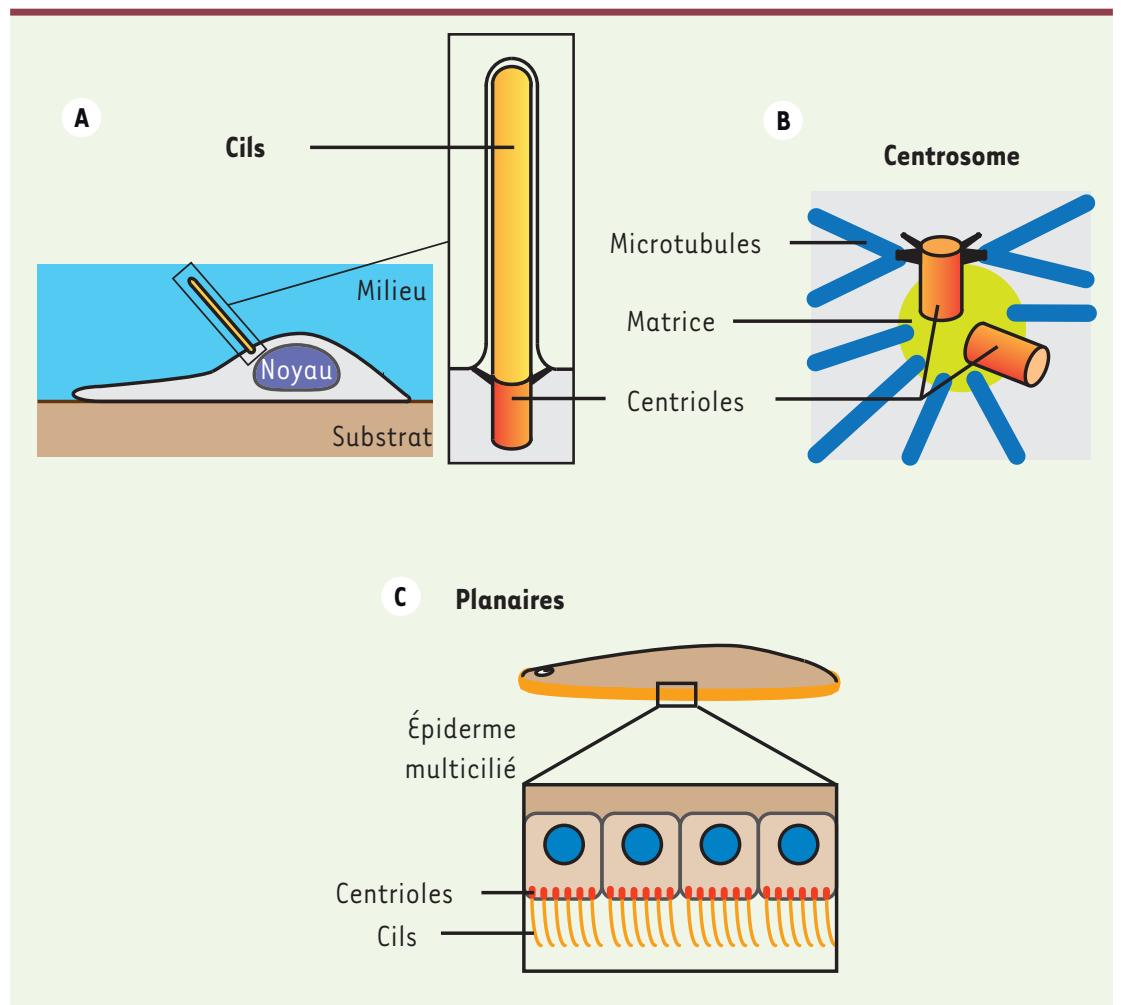

Figure 1. Cils, centrioles et centrosomes. A. Les cils sont des projections cellulaires assemblées à partir des centrioles. Pour une cellule en culture posée sur un substrat, le cil primaire (sensoriel) pointe dans le milieu pour «sentir » l'environnement. $\boldsymbol{B}$. Les centrioles forment aussi le cœur du centrosome, un organite cellulaire qui contrôle l'assemblage et l'organisation spatiale des microtubules, éléments majeurs du cytosquelette. Les microtubules sont formés à partir de la matrice qui entoure les centrioles, qui eux assurent la stabilité et la duplication du centrosome. C. Les planaires se déplacent grâce un épiderme multicilié ventral, dans lequel chaque cellule assemble une centaine de centrioles au cours de la différenciation. La structure de ces centrioles, telle qu'elle apparaît en microscopie électronique, est similaire à celle des centrioles du centrosome humain. 
l'assemblage simultané de nombreux centrioles lors de la différenciation des cellules multiciliées. Chez l'humain, les cellules multiciliées s'organisent en tissus épithéliaux qui bordent les voies respiratoires, les ventricules cérébraux ainsi que les oviductes, et permettent, respectivement, l'évacuation du mucus, la circulation du liquide cérébrospinal et le transport des ovocytes.

Des épidermes multiciliés permettent aussi la locomotion d'animaux de petite taille comme les planaires (Figure IC). Les planaires sont des vers plats, ou plathelminthes, étudiés depuis près de deux siècles pour leur extraordinaire capacité de régénération. Elles peuvent en effet régénérer un organisme entier à partir de très petits fragments issus de pratiquement n'importe quelle partie du corps, grâce à des cellules souches totipotentes appelées néoblastes [4]. Le fait que les planaires utilisent la motilité ciliaire pour leur locomotion permet aussi de détecter des défauts dans l'assemblage des centrioles ou des cils simplement en observant le déplacement des animaux.

Les planaires : un modèle d'étude des cellules multiciliées humaines Grâce aux efforts du laboratoire d'Alejandro Sanchez Alvarado (Stowers Institute, États-Unis), le génome de la planaire Schmidtea mediterranea, ainsi que des méthodes d'inactivation de l'expression de gènes par interférence ARN, sont à présent disponibles [5]. Nous avons donc utilisé les planaires comme modèle pour l'étude des épithéliums multiciliés humains. Notre premier objectif a été d'identifier les mécanismes d'assemblage des centrioles dans les cellules multiciliées. La structure de ces centrioles, telle qu'elle apparaît en microscopie électronique, est similaire à celle des centrioles des cellules en division, et nous avons donc fait l'hypothèse que des composants du centrosome étaient aussi impliqués dans l'assemblage des centrioles des cellules multiciliées. En s'appuyant sur une étude protéomique des composants du centrosome humain [6], nous avons établi une liste de gènes candidats et nous avons ensuite cherché s'ils étaient aussi présents dans le génome de la planaire. Nous avons trouvé des homologues pour la majorité de ces gènes chez S. mediterranea, et nous les avons inactivés par interférence ARN pour déterminer leur fonction. Pour environ $80 \%$ des gènes présents chez la planaire, nous avons observé un défaut de locomotion des planaires, suggérant un problème dans l'assemblage des centrioles dans les cellules multiciliées. Nous avons analysé plus en détail un certain nombre de ces gènes, ce qui nous a permis de caractériser la voie d'assemblage des centrioles dans les cellules multiciliées et d'identifier de nouveaux gènes candidats pour des ciliopathies humaines [7]. Si l'inactivation des homologues de protéines comme Plk4, Sas-4 ou Sas-6, nécessaires à la duplication des centrosomes chez d'autres espèces animales, entraîne des défauts très importants dans la locomotion des planaires, elle n'affecte en revanche pas leur régénération. La régénération nécessite des divisions cellulaires, et l'on aurait pu s'attendre à ce que la perturbation de protéines essentielles à la duplication du centrosome affecte la division des néoblastes et donc la régénération. $\varepsilon$ n fait, nous avons observé que les néoblastes, ainsi que toutes les cellules non ciliées des planaires, ne possèdent pas de centriole. En outre, le gène codant pour une protéine essentielle à la fonction et la duplication du centrosome, appelée SPD-2, est présent dans tous les génomes animaux connus à ce jour, mais absent du génome de la planaire. Ces résultats démontrent que ces animaux ont la particularité de ne jamais assembler de centrosome, et forment des centrioles uniquement au cours de la différenciation des cellules ciliées.

\section{Analyse phylogénétique \\ des centrosomes chez les planaires}

Les planaires dérivent, comme tous les autres animaux, d'un ancêtre qui possédait un centrosome et la protéine SPD-2. Quand, lors de l'évolution des planaires, le centrosome, organite si conservé chez les autres animaux, a-til été éliminé ? Pour tenter de répondre à cette question, nous nous sommes intéressés à une espèce se situant à la base de l'arbre phylogénétique des plathelminthes, appelée Macrostomum lignano, dont le génome a été récemment publié. Contrairement aux planaires, le génome de cette espèce possède bien un homologue du gène $S P D-2$, et une étude réalisée sur une espèce très proche, Macrostomum hystricinum, suggère que des centrosomes sont bel et bien présents dans les cellules en division [8]. Les vers plats du genre Macrostomum et les planaires ont beaucoup de similitudes du point de vue anatomique et les deux groupes sont capables de régénération grâce à des néoblastes. Dès lors, comment expliquer la présence du centrosome dans un cas et pas dans l'autre? Une importante différence réside dans le mode de clivage embryonnaire. Le genre Macrostomum utilise un mode de clivage ancestral appelé clivage spiral, qui repose sur une succession de divisions très stéréotypées en orientation contrôlée (Figure 2). Or, le centrosome est impliqué dans le contrôle de l'orientation des plans de division chez la levure et les animaux [9]. Au contraire, les planaires présentent un mode de clivage dérivé qui n'implique pas de divisions en orientation contrôlée (Figure 2) [10]. C'est aussi le cas des schistosomes, des vers plats parasitaires responsables de la bilharziose qui, eux non plus, ne possèdent pas d'homologues du gène SPD-2 dans leur génome [7].

Le modèle que nous proposons est que la perte du centrosome au cours de l'évolution des planaires, et probablement aussi de celle des vers plats parasitaires, est liée au fait que ces animaux ont mis en place des mécanismes développementaux ne nécessitant pas de divisions en orientation contrôlée. De façon surprenante, l'évolution du centrosome, un 


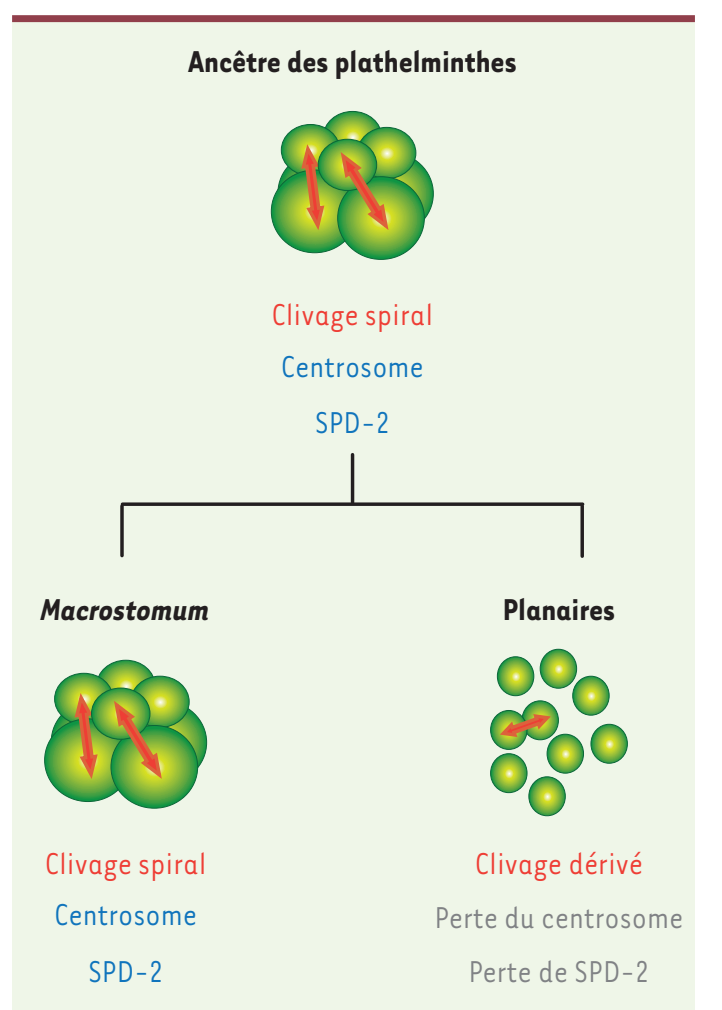

Figure 2. Modèle expliquant la perte du centrosome chez les planaires. Notre hypothèse est que la perte du centrosome est liée à un changement dans le mode de développement embryonnaire qui s'est produit au cours de l'évolution des plathelminthes. L'ancêtre des plathelminthes, ainsi que les plathelminthes qui se situent à la base de l'arbre phylogénétique, comme Macrostomum, utilisent un mode de clivage embryonnaire appelé clivage spiral. Le clivage spiral nécessite un contrôle précis de l'orientation des plans de divisions cellulaires, et donc a priori la présence de centrosomes. Chez les planaires, le développement n'implique pas de divisions en orientation contrôlée, et le centrosome a été éliminé.

organite déjà présent chez nos ancêtres unicellulaires, semble donc être essentiellement corrélée chez les animaux à des processus de développement, et pas seulement à des fonctions strictement confinées à chaque cellule.

L'étude des planaires nous renseigne donc, non seulement sur l'assemblage des centrioles et des cils affectés dans nombre de ciliopathies, mais aussi sur la fonction, la composition et l'évolution du centrosome, précisément parce que ce dernier a été perdu au cours de l'évolution des planaires. $\Delta$

What planarians tell us about cilia, centrioles and centrosomes

NOUVELLE

\section{Une altération de la neurogenèse conduit à des troubles de l'olfaction dans la sclérose en plaques}

Vanja Tepavčević, Brahim Nait-Oumesmar, Anne Baron-Van Evercooren
LIENS D'INTÉRÊT

Les auteurs déclarent n'avoir aucun lien d'intérêt concernant les données publiées dans cet article.

\section{REMERCIEMENTS}

L'auteur remercie Nicolas Borghi pour ses commentaires sur le manuscrit. Ce travail a été soutenu par le National Institutes of Health (NIH) et la Fondation W.M. Keck.

\section{RÉFÉRENCES}

1. Goetz SC, Anderson KV. The primary cilium: a signalling centre during vertebrate development. Nat Rev Genet 2010 ; 11 : 331-44.

2. Nigg $\varepsilon A$, Raff JW. Centrioles, centrosomes, and cilia in health and disease. Cell $2009 ; 139: 663-78$.

3. Azimzadeh J, Marshall WF. Building the centriole. Curr Biol $2010 ; 20$ : R816-25.

4. Wagner DE, Wang $I \varepsilon$, Reddien PW. Clonogenic neoblasts are pluripotent adult stem cells that underlie planarian regeneration. Science 2011 ; 332 : 811-6.

5. Newmark PA, Reddien PW, Cebria F, Sanchez Alvarado A. Ingestion of bacterially expressed double-stranded RNA inhibits gene expression in planarians. Proc Natl Acad Sci USA 2003; 100 : 11861-5

6. Andersen JS, Wilkinson CJ, Mayor T, et al. Proteomic characterization of the human centrosome by protein correlation profiling. Nature $2003 ; 426: 570-4$.

7. Azimzadeh J, Wong ML, Downhour DM, et al. Centrosome loss in the evolution of planarians. Science 2012 ; 335 : 461-3.

8. Tyler S. Development of cilia in embryos of the turbellarian Macrostomum. Hydrobiologia 1981 ; $84: 231-9$

9. Pereira G, Yamashita YM. Fly meets yeast: checking the correct orientation of cell division. Trends Cell Biol $2011 ; 21: 526-33$.

10. Cardona A, Hartenstein V, Romero R. Early embryogenesis of planaria: a cryptic larva feeding on maternal resources. Dev Genes Evol 2006 ; 216 : 667-81.
Centre de recherche de l'institut du cerveau et de la moelle épinière (Cricm), université Pierre et Marie Curie (UPMC) Paris 6, UMR-S975, Inserm U975, CNRS UMR 7225, assistance publique-hôpitaux de Paris (AP-HP), hôpital Pitié-Salpêtrière, 47, boulevard de l'Hôpital, 75013 Paris, France.

anne.baron@upmc.fr

[1] et participent, directement ou indirectement, à la ségrégation spatiale des réseaux neuronaux [2]. Les cellules souches de la ZSV assurent le renouvellement des interneurones et neurones périglomérulaires du bulbe olfactif dont
Les cellules souches du gyrus denté et de la zone sous-ventriculaire Ces dernières décennies ont été marquées par la découverte de cellules souches (CS) dans certaines régions du cerveau adulte: le gyrus denté et la zone sous-ventriculaire (ZSV). Ces cellules donnent naissance, tout au long de la vie, à des neurones aux fonctions particulières: les neurones issus des cellules souches du gyrus denté s'intègrent dans les circuits propres à cette structure 